\title{
GENERALISED DISTANCES OF SEQUENCES I: $B$-DISTANCES
}

\author{
BENEDEK NAGY
}

Received 15 June, 2016

\begin{abstract}
In this paper, we investigate the $B$-distances of infinite sequences. For this purpose we use generalized neighbourhood sequences. The general neighbourhood sequences were introduced for measuring distances in digital geometry $\left(\mathbb{Z}^{n}\right)$. We extend their application to sequences, and present an algorithm which provides a shortest path between two sequences. We also present a formula to calculate the $B$-distance of any two sequences for a neighbourhood sequence $B$.

We also investigate the concept of $k$-convergent sequences for $k \in \mathbb{N}$, that concept is generally weaker than the convergence. We will use the term $k$-sequence which is a kind of generalization of the concept of 0 -sequence. We also show some connection between the $B$-distances of sequences and the properties of their difference sequences.
\end{abstract}

2010 Mathematics Subject Classification: 11Y55; 40A05; 52C07; 68U10

Keywords: sequences, neighbourhood sequences, distance, metric space, geometry of sequences

\section{INTRODUCTION}

The theory of sequences is widely used in mathematical analysis, calculus and in various applications. A good old textbook in this topic is Knopp's book [8].

One of the first used distance among finite sequences was the Hamming-distance [1,3]: the $H$-distance of two same-length sequences over a finite alphabet is the number of places where they differ. We can extend this definition to infinite sequences also, over infinite alphabets $(\mathbb{Z}$, or $\mathbb{R})$. Allowing infinite distances this extension is natural.

There are other possibilities to measure the distances of finite and infinite sequences which contain numbers. The supremum norm is used for example in [9]. We will call this metric as sup-distance. It is another possibility to use the inf-distance, which is not a widely used distance function (due to some unpleasant properties).

In this paper, we investigate distances with integer values, they are based on various neighbourhood sequences $(B)$ and neighbouring relations among the sequences.

The theory of neighbourhood sequences comes from digital geometry. In digital geometry the discrete space is used, i.e., points can have only integer co-ordinates. 
Two different points in $\mathbb{Z}^{m}$ are $k$-neighbours $(k, m \in \mathbb{N} \cup\{\infty\}, k \leq m)$, if their corresponding elements are equal up to at most $k$ exceptions, and the difference of the exceptional values are at most 1 . While in digital geometry the elements of $\mathbb{Z}^{n}$ or $\mathbb{Z}^{\infty}$ were called points, we will call the elements of $\mathbb{R}^{\infty}$ sequences. Indeed, we can use the same neighbourhood criteria for sequences.

After fixing the value of $k$, we may define the distance of two sequences as the number of steps of the shortest path between these sequences, where a step means a movement from a sequence to one of its $k$-neighbours. On can check that by this definition we get a generalized metric on $\mathbb{R}^{\infty}$, for each $k \in\{1,2, \ldots\}$, and that these generalized metrics are different for the separate values of $k$.

To obtain these distances we fixed $k$ in the beginning, in other words, we used the same $k$ in each step for walking from a sequence $p$ to a sequence $q$ in $\mathbb{R}^{\infty}$. The situation is more complicated if we may change the value of $k$ after every step. A sequence $\left(b_{i}\right)_{i=1}^{\infty}$ is called a neighbourhood sequence over the set of the sequences, if $b_{i} \in \mathbb{N} \cup\{\infty\}(i \in \mathbb{N})$. The concept of distances based on neighbourhood relations comes from $[6,13]$. The periodic neighbourhood sequences were introduced in $[5$, $6,14]$, while the general notion in $[7,10]$. (We mention that the sequences in $[5,14]$ were called "neighbourhood sequences" while in [7] "generalized neighbourhood sequences", but for simplicity we use the above definition.) Moreover in [7, 10,11] the authors investigated the $\infty$-dimensional space $\mathbb{Z}^{\infty}$.

By the help of an neighbourhood sequence $\left(b_{i}\right)_{i=1}^{\infty}$ we may define the distance of sequences $p, q$ in the following way. We take the length of a shortest path from $p$ to $q$, but at the $i$-th step, now, we may move from a sequence to another if and only if they are $b_{i}$-neighbours. Certainly, this notion is a generalization of the original one, as we may choose $b_{i}=k$ for each $i \in \mathbb{N}$, with any $k \in\{1,2, \ldots\}$. It is obvious, that these so-called $B$-distances have only non-negative integer values based on their definitions.

In [10] we have presented an algorithm which provides a shortest path between digital points, both in case of finite and infinite dimensional spaces. As we mentioned, the neighbourhood sequence $\left(b_{i}\right)_{i=1}^{\infty}$ with $b_{i}=k(i \in \mathbb{N})$ generates a generalized metric on $\mathbb{R}^{\infty}(m \in \mathbb{N})$ for any $k$. However, it is easy to find neighbourhood sequences, even periodic ones, such that the distances with respect to these neighbourhood sequences do not provide (generalized) metrics on the set of sequences. In [10] we have proved a necessary and sufficient condition for distance functions based on neighbourhood sequences to define a generalized metric in $\mathbb{Z}^{\infty}$. The purpose of this paper is to generalize these concepts from $\mathbb{Z}^{\infty}$ to $\mathbb{R}^{\infty}$.

The structure of this paper is as follows. In the next section, we give our notation and we define basic concepts. In the third section, we present an algorithm to solve the shortest path problem between any two sequences. In section four, we describe some properties of $B$-distances. We compare the $B$-distances with other type of distances. We also present a necessary and sufficient condition to a $B$-distance to 
give a generalized metric over the set (i.e., the space) of sequences. A formula to calculate the $B$-distance between two arbitrary sequence with a given neighbourhood sequence $B$ is also derived.

Although some of the concepts corresponding to sequences and distances are wellknown we briefly recall them; we believe that this general overview will be helpful to understand the subsequent parts of the paper. Some of the results may seem to be simple to follow, but we have decided to include them here to have a self-contained paper and to show a relatively complete picture about this field.

\section{NOTATIONS AND DEFINITIONS}

First, we recall and define some basic concepts about the sequences. Although some of the concepts are well known from the literature mentioned earlier, we believe that due to various relations of these concepts to our topic it is worth to recall them in details. We start with the simplest concepts and we are going to the direction of more complex concepts. Let us start with a notation. Throughout the paper $\mathbb{R}^{\infty}$ will denote the set of all sequences. A sequence $p=(p(i))_{i=1}^{\infty}$ is periodic if there is a value $l \in \mathbb{N}$ such that $p(i)=p(i+l)$ for each element of $p$ ( $l$ is called the period of p).

Definition 1. A sequence $p=(p(i))_{i=1}^{\infty}$ - where $p(i) \in \mathbb{R}$ for all $i$-is convergent if there exists $x \in \mathbb{R}$ such that for all $\varepsilon>0$ there is an $n(\varepsilon)$ such that for all $n \in \mathbb{N}$ if $n>n(\varepsilon)$, then $|p(n)-x|<\varepsilon$. We say that $x$ is the limit of the sequence $p$. If a sequence convergent and its limit is 0 then we call it 0 -sequence.

In this paper, we investigate more general types of convergence: $k$-convergences.

Definition 2. A sequence $p \in \mathbb{R}^{\infty}$ is $k$-convergent (for a fixed non-negative value $k$ ), if there exists $n \in \mathbb{N}$ such that for all $i, j$ with $i>n$ and $j>n$ we have $\mid p(i)-$ $p(j) \mid \leq k$.

Now we extend the definition of 0 -sequence to $k$-sequence in the following way.

Definition 3. A sequence $p \in \mathbb{R}^{\infty}$ is $k$-sequence (for a fixed positive value of $k$ ) if there exists a natural number $n$ such that for all $i \in \mathbb{N}$ if $i>n$ then $|p(i)|<k$.

The next statements are evident about the relation of convergence, $k$-convergence and $k$-sequence.

\section{Proposition 1.}

1.1 If a sequence $p$ is $k$-convergent, then it is $k^{\prime}$-convergent for all $k^{\prime}>k$.

1.2 The sequence $p$ is convergent if and only if it is $k$-convergent for all $k>0$.

1.3 The sequence $p$ is 0 -convergent if and only if its tail is constant. (In this case it is convergent, also.)

1.4 If the sequence $p$ is a $k$-sequence for some $k>0$, then it is $2 k$-convergent. Moreover, if $p(i) \geq 0$ for all $i \in \mathbb{N}$, then $p$ is also $k$-convergent. 
1.5 For each $k$-convergent sequence $p$ there is a value $j$ such that, $p$ is $j$-sequence. 1.6 The (elementwise) sum and the (elementwise) difference of a $k$-convergent sequence $p$ and $a j$-convergent sequence $q$ are $k+j$-convergent.

Lemma 1. If the sequences $p$ and $q$ are convergent, then their sum-and their difference-sequence are $k$-convergent for some $k$.

Proof. It is a simple consequence of the facts in Proposition 1 (and the previous definitions).

For periodic sequences we have the following facts.

\section{Proposition 2.}

2.1 Each periodic sequence $p$ with period $l$ is $k$-convergent for $k \geq \max _{1 \leq i, j \leq l}(p(i)-p(j))$.

2.2 Each periodic sequence $p$ with period $l$ is $k$-sequence for $k \geq \max _{1 \leq i \leq l}(|p(i)|)$.

From here we will use the terms $k$-convergence and $k$-sequence with arbitrary non-negative integer values of $k$, however our definition works for all (not necessary integer) non-negative value of $k$.

Now, we give some basic ideas about our distance functions.

Definition 4. A function $d: \mathbb{R}^{\infty} \times \mathbb{R}^{\infty} \rightarrow \mathbb{R} \cup\{\infty\}$ is called a generalized metric on $\mathbb{R}^{\infty}$, if it satisfies the following conditions:

- a) $\forall p, q \in \mathbb{R}^{\infty}: d(p, q) \geq 0$, and $d(p, q)=0$ if and only if $p=q$ (positive definiteness),

- b) $\forall p, q \in \mathbb{R}^{\infty}: d(p, q)=d(q, p)$, (symmetry)

- c) $\forall p, q, r \in \mathbb{R}^{\infty}: d(p, q)+d(q, r) \geq d(p, r)$ (triangle inequality).

Moreover, if for every possible pair of $p, q \in \mathbb{R}^{\infty}$ the distance $d(p, q)$ is finite, then it is a metric.

If instead of point $a$ ) we have only

- $\left.a^{\prime}\right) \forall p, q \in \mathbb{R}^{\infty}: d(p, q) \geq 0$, and $d(p, p)=0$,

then the function $d: \mathbb{R}^{\infty} \times \mathbb{R}^{\infty} \rightarrow \mathbb{R} \cup\{\infty\}$ is a semi-metric on $\mathbb{R}^{\infty}$.

For measuring distances of sequences, usually the so-called supremum norm is used [9]. We can use also the Hamming-distance; it is finite only when the two sequences differ in finitely many places. The $H$-distance is one of the first discrete distances, it results always only non-negative integer values.

Definition 5. The sup-distance of the sequence $p$ and $q$ is given by

$$
d(p, q ; \text { sup })=\sup (|p(i)-q(i)|) .
$$


The Hamming-distance of $p$ and $q$ is

$$
d(p, q ; H)=\sum_{p(i) \neq q(i)} 1
$$

The inf-distances of $p$ and $q$ is

$$
d(p, q ; \inf )=\inf (|p(i)-q(i)|) .
$$

The discrete metric over the set of sequences is the following:

$$
d(p, q ; d i s c)= \begin{cases}0, & \text { if } p=q \\ 1, & \text { if } p \neq q .\end{cases}
$$

It is well-known that $d(p, q ;$ sup $)$ and $d(p, q ; H)$ are generalized metrics over $\mathbb{R}^{\infty}$. For the inf-distance one can prove that the properties b) and a') of Definition 4 hold only, therefore it is an unusual distance. The discrete metric is the simplest metric, but it is not practical for applications.

One of our most important investigations is introducing the neighbourhood relation among sequences.

Definition 6. Let $p$ and $q$ be two sequences in $\mathbb{R}^{\infty}$. Let $k$ be a non-negative integer. The sequences $p$ and $q$ are $k$-neighbours, if the following two conditions hold:

- $|p(i)-q(i)| \leq 1$ for all $i \in \mathbb{N}$, and

- $\sum_{i \in \mathbb{N}, p(i) \neq q(i)} 1 \leq k$.

Definition 7. The infinite sequence $B=\left(b_{i}\right)_{i=1}^{\infty}\left(b_{i} \in \mathbb{N} \cup\{\infty\}\right)$ is called a neighbourhood sequence. If for some $l \in \mathbb{N}, b_{i}=b_{i+l}$ holds for every $i \in \mathbb{N}$, then $B$ is called periodic (with period $l$ ).

For investigating distances of sequences, we will use their difference sequences in the following way.

Notation 1. Let $p$ and $q$ be two sequences. Put $w(i)=|p(i)-q(i)|$ for all $i$, and $w=(w(i))_{i=1}^{\infty}$. The sequence $w$ is called the (absolute) difference of $p$ and $q$.

The up-integer-difference-sequence (uids) $u$ of $p$ and $q$ is defined by the top (i.e., ceiling) of the elements of their absolute difference as $u(i)=\lceil w(i)\rceil=\lceil\mid p(i)-$ $q(i) \mid\rceil$, where $\lceil x\rceil$ is the upper integer part of the real number $x$, i.e. $\lceil x\rceil=\inf \{k \mid k \in$ $\mathbb{Z}, k \geq x\}$.

Definition 8. Let $p$ and $q$ be two sequences and $B=\left(b_{i}\right)_{i=1}^{\infty}$ be an neighbourhood sequence. A finite sequence of sequences $\Pi(p, q ; B)$ of the form $p=p_{0}, p_{1}, \ldots, p_{m}=$ $q$, where $p_{i-1}, p_{i} \in \mathbb{R}^{\infty}$ are $b_{i}$-neighbours for $1 \leq i \leq m$, is called a $B$-path from $p$ to $q$. We write $m=|\Pi(p, q ; B)|$ for the length of the path. 
Remark 1. It is possible that there are no $B$-paths between two sequences. For example, if the set $\{|p(i)-q(i)|: i \in \mathbb{N}\}$ - i.e. the set of elements of the difference sequence of them - is unbounded, then there are no neighbourhood sequence $B$, for which a $B$-path would exist between the sequences $p=(p(i))_{i=1}^{\infty}$ and $q=(q(i))_{i=1}^{\infty}$.

In the next section we will prove a necessary and sufficient condition for the existence of $B$-path between two sequences.

Now, we are ready to define the $B$-distance of any two sequences.

Definition 9. Let $p, q \in \mathbb{R}^{\infty}$ and $B$ be an neighbourhood sequence. If there is no $B$-path between $p$ and $q$, then we put $d(p, q ; B)=\infty$. Otherwise, denote by $\Pi^{*}(p, q ; B)$ a shortest path (i.e., a $B$-path with minimal length) from $p$ to $q$, and set $d(p, q ; B)=\left|\Pi^{*}(p, q ; B)\right|$. We call $d(p, q ; B)$ the $B$-distance of the sequences $p$ and $q$.

It is evident that using the definition of $B$-distance above, it is positive definite (point a) of Definition 4) for any neighbourhood sequence $B$.

Definition 10. Let $B_{1}$ and $B_{2}$ be two neighbourhood sequences. We say that $B_{1}$ is faster than $B_{2}$, if

$$
d\left(p, q ; B_{1}\right) \leq d\left(p, q ; B_{2}\right) \quad \text { for all } p, q \in \mathbb{R}^{\infty} .
$$

We denote this relation by $B_{1} \sqsupseteq^{*} B_{2}$.

Originally, the relation $\sqsupseteq^{*}$ was introduced by Das [4] in the two dimensional digital space, and by Fazekas et al. in [7] for higher dimensions. We will use it for infinite sequences $\left(\mathbb{R}^{\infty}\right)$.

For later use we need to introduce some further notations.

Definition 11. Let $m \in \mathbb{N}$ and $B=\left(b_{i}\right)_{i=1}^{\infty}$ an neighbourhood sequence. Put

$$
b_{i}^{(m)}=\min \left(b_{i}, m\right) \text { and } B^{(m)}=\left(b_{i}^{(m)}\right)_{i=1}^{\infty} .
$$

The sequence $B^{(m)}$ is called the $m$-limited sequence of $B$. Denote by $f_{k}(i)$ the $i$-th subsums of the $k$-limited sequence of $B$, i.e., put

$$
f_{k}(i)=\left\{\begin{aligned}
\sum_{j=1}^{i} b_{j}^{(k)}, & \text { if } i \geq 1, \\
0, & \text { if } i=0 .
\end{aligned}\right.
$$

Definition 12. Let $B=\left(b_{i}\right)_{i=1}^{\infty}$ be an neighbourhood sequence. The sequence $B(j)=\left(b_{i}\right)_{i=j}^{\infty}$ is called the $j$-shifted neighbourhood sequence of $B$. 


\section{Minimal PATH}

In this section, we give an algorithm which provides a shortest path between arbitrary two sequences, if such a path exists. As we mentioned in Remark 1, it is possible that there is no path between two given sequences with a given neighbourhood sequence. The following lemma provides a criterion for the existence of a path between two sequences.

Lemma 2. A finite B-path exists between the sequences $p$ and q, i.e. their distance is finite, if and only if the difference sequence of them ( $w$ ) has a supremum and the neighbourhood sequence $B$ contains the symbol $\infty$, at least $k$ times, where $k$ is the maximal element of the uids $(u)$ of the sequences $p$ and $q$, which occurs infinitely many times.

Proof. First, we prove the case when the distance is infinite.

If the difference sequence $w$ of $p$ and $q$ has not got a supremum (like the sequence of natural numbers $(1,2,3, \ldots)$ ), then it is impossible to reach $q$ from $p$ (or from $p$ to $q$ ) in finitely many steps, because if we want to take number $m$ steps from $p$, for every $m \in \mathbb{N}$, we can find an element $i$ of the sequence $w$, such that $w(i)>m$.

If the sequence $B$ contains the symbol $\infty$ less times than $k$, then after the step with $b_{i}$, for which $\forall j \geq i, b_{j}<\infty$, we have infinite number of non-zero values in the difference sequence of $q$ and the reached sequence. Thus, it is impossible to reach the sequence $q$ in finitely many steps, with steps which are changing only finite number of elements.

Now, we are proving the sufficiency of the conditions to have a finite distance:

If the uids $u$ of the sequences $(p, q)$ has a largest element, then we denote this value by $m$. Let the sequence $B$ contain the symbol $\infty$ at least $k$ times. Then after the step with $b_{j}=\infty$, where $b_{j}$ is the $k$-th value $\infty$ in $B$, we have only finite number of non-zero elements in the difference sequence of $q$ and the reached sequence $r$. Forasmuch $m$ was the largest element of the $w$, then after the $j$-th step, each element of the difference sequence of $q$ and $r$ is less or equal to $m$. Thus, we have finitely many finite numbers in the difference of $q$ and $r$, then the sum of them is also finite. Therefore, we need only finitely many steps to reach $q$ from $p$. (Our proof is same from $q$ to $p$.)

Remark 2. If the neighbourhood sequence $B$ is periodic, and it contains the element $\infty$, then $B$ contains $\infty$ at infinitely many positions.

The following algorithm provides one of the shortest $B$-paths between two arbitrary sequences, if such a path exists. This algorithm is based on the algorithm in [10], which works in finite and infinite dimensional digital spaces. (For algorithm works in finite dimensional digital space with only periodic neighbourhood sequences see [5].) 


\section{Algorithm 1}

Input: An neighbourhood sequence $B=\left(b_{i}\right)_{i=1}^{\infty}$ and $p, q \in \mathbb{R}^{\infty}$, such that $d(p, q ; B)<$ $\infty$

- step 1. Let $w^{(0)}$ be the absolute difference of $p$ and $q, t(i)=\operatorname{sgn}(p(i)-$ $q(i)),(i \in \mathbb{N})$, and put $j=0$ and $\Pi=(p)$.

- step 2. If $w^{(j)}(i)=0$ for every $i$ then goto step 8 , else set $j=j+1$.

- step 3. Put $w^{(j)}=w^{(j-1)}$.

- step 4. If $b_{j}$ is finite, then select the largest $b_{j}$ entries of $w^{(j)}$. If $b_{j}$ is infinite, then select all the entries of $w^{(j)}$.

- step 5. For each selected $w^{(j)}(i)$ if $w^{(j)}(i) \geq 1$, let $w^{(j)}(i)=w^{(j-1)}(i)-1$ else let $w^{(j)}(i)=0$.

- step 6. Append to the path $\Pi$ the sequence $x_{j}$ defined by $x_{j}(i)=q(i)+$ $w^{(j)}(i) t(i)$ for all $i$.

- step 7. Goto 2 .

- step 8. Output $\Pi$ as a minimal $B$-path between $p$ and $q$, and $j$ as the length of this path.

Lemma 3. The B-distance of the sequences $p$ and $q$ is invariant to the shift, i.e. for arbitrary $r \in \mathbb{R}^{\infty}$ the following statement is true:

$$
d(p, q ; B)=d\left((p(i)+r(i))_{i=1}^{\infty},(q(i)+r(i))_{i=1}^{\infty} ; B\right) .
$$

Proof. If $d(p, q ; B)$ is finite, then let $\Pi^{*}(p, q ; B):\left(p=p_{0}\right), p_{1}, \ldots,\left(p_{m}=q\right)$ be a shortest path from $p$ to $q$.

It is easy to check that $\Pi\left(p^{\prime}, q^{\prime} ; B\right)$ with sequences $p_{j}^{\prime}=\left(p_{j}(i)+r(i)\right)_{i=1}^{\infty}(0 \leq$ $j \leq m)$ is also a shortest $B$-path between $p^{\prime}=(p(i)+r(i))_{i=1}^{\infty}$ and $q^{\prime}=((q(i)+$ $r(i))_{i=1}^{\infty}$.

Otherwise, suppose that $d(p, q ; B)=\infty$. Our aim to show that $d\left(p^{\prime}, q^{\prime} ; B\right)=\infty$ also with a shift by the sequence $r$. Contrary suppose that it is not. Then, we have a shortest path between $p^{\prime}$ and $q^{\prime}$. Now it is finite distance therefore using the previous part of the proof we have the same distance between $p^{\prime \prime}$ and $q^{\prime \prime}$ where we use a shift by an arbitrary $r^{\prime}$. We choose $r^{\prime}$ as the sequence $(-r(i))_{i=1}^{\infty}$, therefore $p^{\prime \prime}=p$ and $q^{\prime \prime}=q$. But it is a contradiction, because a distance is finite or infinite, but it is not both of them.

Observe that the algorithm is a greedy algorithm. The following theorem is about the correctness of our algorithm. We use the term step as step of the algorithm, however some steps are complex.

Theorem 1. Algorithm 1 terminates after finitely many steps and provides a Bpath with minimal length between the sequences $p$ and $q$. 
Proof. We will deduce the statement to the case of sequences over $\mathbb{Z}^{\infty}$, for which similar theorem was proved in [10].

Using Lemma 3, without loss of generality, we can assume that the sequence $p=$ $(0)_{i=1}^{\infty}$ and $q$ is an arbitrary element of $\mathbb{R}^{\infty}$.

It is evident that our distance is based on the number of steps, independently how much is the change of the values in a step (i.e., they are 1, or may be less than 1). Substituting the values which changes less than 1 via the values are changing exactly 1 , we get the same distance.

Therefore, the distance of arbitrary two sequences can be calculated by

$$
d(p, q ; B)=d(o, u ; B),
$$

where $o=(0)_{i=1}^{\infty}$ and $u$ is the uids of $p$ and $q$.

The sequences $o$ and $u$ contain only integer values, therefore the correctness of the algorithm follows from the correctness of the algorithm working on sequences in $\mathbb{Z}^{\infty}[10]$.

Lemma 4. The B-distance is symmetric.

Proof. Our proof is based on the algorithm. In the case when at step 6 we append the sequence $x_{j}(i)=p(i)-w^{(j)}(i) t(i)$ for all $i$ we get a shortest path from $q=x_{0}$ to $p$, which has the same length as the shortest path from $p$ to $q$. In the case of infinite distance: if there is no shortest path between two sequences, then it does not exist in any directions.

Consequence 1. The $B$-distance of two sequences $p$ and $q$ depends only on the neighbourhood sequence $B$ and the up-integer-difference-sequence $u$ of $p$ and $q$.

Using Consequence 1 we can adopt some properties from [10], where only the sequences with integer values was studied. In the next section we analyse some properties of the $B$-distances.

\section{Properties of $B$-Distances of SEQuences}

In this section, first we state the relation between the sup-distance and the $B$ distances.

Proposition 3. For any two $p, q \in \mathbb{R}^{\infty}$,

$$
d\left(p, q ;(\infty)_{i=1}^{\infty}\right)=\lceil d(p, q ; \text { sup })\rceil .
$$

Remark 3. Using a variation of Algorithm 1 and the definition of $B$-distances with $B=(\infty)_{i=1}^{\infty}$ we get exactly the same value for this, $(\infty)$-distance, as for the sup-distance. The difference should be at the last step by reaching $q$. Increasing the path length by $\sup \left(w^{(j-1)}(i)\right)$ instead of 1 in step 2 of the algorithm the provided minimal path-length will be exactly the same as the sup-distance of $p$ and $q$. 
Proposition 4. Using the neighbourhood sequence (1) ${ }_{i=1}^{\infty}$ the distance (we will use the notation (1)-distance) of any two sequences $p$ and $q$ is at least the number of elements in which they differ, i.e.

$$
d\left(p, q ;(1)_{i=1}^{\infty}\right) \geq \sum_{p(i) \neq q(i)} 1=d(p, q ; H) .
$$

Moreover, we have the following statement among the values of $B$-distances.

Lemma 5. For any two sequence $p$ and $q$ the distances are in the following relation:

$$
\sum\lceil u(i)\rceil=d\left(p, q ;(1)_{i=1}^{\infty}\right) \geq d(p, q ; B) \geq d\left(p, q ;(\infty)_{i=1}^{\infty}\right)=\max (\lceil u(i)\rceil),
$$

where $B$ is an arbitrary neighbourhood sequence.

Proof. It is easy to show by using the algorithm.

Using neighbourhood sequences for calculating distances between sequences we have wide a variety of distances between the distances using $(1)_{i=1}^{\infty}$ and $(\infty)_{i=1}^{\infty}$. These two distances are the ceiling (i.e., top) of the distances $L_{1}$ and $L_{\infty}$, respectively (see [2]). Although the $L$-distances (also known as Minkowski distances) were originally introduced for points, we can define and use them for infinite sequences also: $d\left(p, q ; L_{1}\right)=\sum w(i)$ and $d\left(p, q ; L_{\infty}\right)=\sup (w(i))$, the distances $L_{j}$ is defined by $\left(\sum(w(i))^{j}\right)^{\frac{1}{j}}$, the distance $L_{2}$ is the usual Euclidean distance. The distance $L_{\infty}$ is given by the limit of distances $L_{i}$ with $i \rightarrow \infty$ and it is the same as the sup-distance. These $L$-distances are not pleasant in many cases because for infinite sequences the sum $\sum_{i}(w(i))^{j}$ is usually infinite. Hence calculating the $L$-distances we have some difficulties. (In [12] we return to this problem.) For these reasons we recommend the $B$-distances which have more pleasant properties in this point of view.

The previous lemma is about the values of $B$-distances. We will study more precisely the relation 'faster' of neighbourhood sequences later.

Lemma 6. The following statements are equivalent.

- The B-distance of $p, q \in \mathbb{R}^{\infty}$ is finite for every neighbourhood sequence $B$.

- The sequences $p$ and $q$ have the same tail (i.e. $\exists j, \forall k>j, p(k)=q(k)$ ).

- The difference sequence $w$ of $p$ and $q$ is 0 -convergent and 0 -sequence.

Proof. It is evident from the definitions that a sequence has only finitely many nonzero elements if and only if it is 0 -convergent and 0 -sequence. (And the difference sequence of the sequences $p$ and $q$ has this property if and only if they have the same tail.) Otherwise, from Lemma 5 we know that the (1)-distance is the greatest among the $B$-distances. Moreover the (1)-distance of two sequences - for which their difference sequence has only finitely many non-zero element - is finite. Therefore every $B$-distance of them is finite, thus the equivalence of the statements follows. 
Moreover, we generalize the fact above in the following theorem.

Theorem 2. The B-distance of two arbitrary sequences $p$ and $q$ is finite with the neighbourhood sequence $B$ including the symbol $\infty$ at least $k$ times if and only if the difference $w$ of $p$ and $q$ is a $k$-sequence. (And their $B$-distance is infinite if the number of $\infty$ is $k$ in $B$, and the difference $w$ is not a $k$-sequence.)

Proof. If the difference sequence $w$ of $p$ and $q$ is a $k$-sequence, then it does not contain greater values than $k$ infinitely many times (its tail does not contain such elements). Obviously, applying the algorithm to obtain a shortest path, after the $k$-th step by the elements $\infty, w$ contains only finitely many non-zero elements, and their sum is finite, hence each of them is finite. Therefore, after the step by the $k$-th $\infty$, we need only finitely many steps to reach $q$. Thus, it is proven that if $B$ contains at least $k$-times the symbol $\infty$, then the $B$-distance of $p$ and $q$ is finite when their difference is a $k$-sequence. In the other way around, if $w$ is not a $k$-sequence, then it must contain infinitely many elements which are not less than $k$. In this case, after the last $\left(k\right.$-th) step with the symbol $\infty$, the sum $\sum_{w^{(j)}(i)>0} 1$ is infinite, therefore we cannot decrease it to 0 with finitely many 'finite'-steps. Therefore, in that case, the $B$-distance of $p$ and $q$ is infinite.

Now we present a formula to calculate the $B$-distance of any two sequences. Using Consequence 1 we adopt the $B$-distance on $\mathbb{Z}^{\infty}$, which can be found in [11]. For this calculation we will use the sequence $v$ which has the same elements as the uids $u$, sorting by non-decreasing order (i.e., the multiset of elements $u$ is the same as the multiset of elements of $v$ and $v(i) \geq v(j)$ for $i<j)$. We also use the $j$-limited sequence $B^{(j)}$ of the neighbourhood sequence $B$ from Definition 11 .

Proposition 5. The B-distance of any two sequences $p$ and $q$ is given by

$$
d(p, q ; B)=\max _{i \in \mathbb{N}}\left\{d^{(i)}(p, q)\right\},
$$

where

$$
d^{(i)}(p, q)=\max \left\{h \mid \sum_{k=1}^{i} v(k)>\sum_{k=1}^{h-1} b_{k}^{(i)}\right\} .
$$

The following lemma is very useful if we would like to decide numerically whether an neighbourhood sequence is faster or not than another one. Here the subsums of neighbourhood sequences of Definition 11 are also used.

Lemma 7. Let $B_{1}$ and $B_{2}$ be two neighbourhood sequences. Then,

$$
d\left(p, q ; B_{1}\right) \leq d\left(p, q ; B_{2}\right), \quad \text { for all } p, q \in \mathbb{R}^{\infty},
$$

if and only if

$$
f_{k}^{(1)}(i) \geq f_{k}^{(2)}(i), \quad \text { for all } i, k \in \mathbb{N},
$$


where $f_{k}^{(1)}(i)$ and $f_{k}^{(2)}(i)$ correspond to $B_{1}$ and $B_{2}$, respectively.

Proof. This statement is given by a simple calculation from the previous proposition. In Proposition 5 we use the values $f_{i}(h-1)$ on the right hand side of the inequalities of the calculation of the values $d^{(i)}(p, q)$. Substituting it to the form for calculating $B$-distance, one can easily check the statement of this lemma.

Remark 4. As a simple consequence of the previous lemma we obtain that if $B_{1}$ and $B_{2}$ are two neighbourhood sequences with $B_{1} \sqsupseteq^{*} B_{2}$, then for every $i \in \mathbb{N}$ among the first $i$ elements of $B_{1}$ there are at least as many $\infty$ symbols as among the first $i$ elements of $B_{2}$.

Based on the previous lemma we study the relation 'faster' among the neighbourhood sequences.

Lemma 8. The faster relation is not a complete ordering, i.e., there are neighbourhood sequences which are non-comparable.

Proof. Let $B_{1}=(\infty, 1,1,1, \ldots)$ (where each element is 1 after the first) and $B_{2}=$ $(10,10, \infty, \infty, \ldots)$ (each element is $\infty$ after the second). Let $p=\left(\frac{1}{i}\right)_{i=1}^{\infty}$ and $q=$ $\left(\frac{2}{i}\right)_{i=1}^{\infty}$; then $d\left(p, q, B_{1}\right)=1$ and $d\left(p, q, B_{2}\right)=3$. Let $r=\left(\max \left(\frac{6}{i}, \frac{1}{2}\right)\right)_{i=1}^{\infty}$, then $d\left(r, q, B_{2}\right)=4$ and $d\left(r, q, B_{1}\right)=6$.

Remark 5. It is clear that $\sqsupseteq^{*}$ is a reflexive, antisymmetric, transitive relation on the set of neighbourhood sequences, i.e., it is a a partial order. However, it does not form a lattice. (It can be proven in the same way as in [7].)

Now, we want to know whether a $B$-distance is a generalized metric above the set of sequences $\mathbb{R}^{\infty}$. The distance based on an arbitrary neighbourhood sequence, in general, does not satisfy the conditions of a (generalized) metric. However, in geometry those distances are the most useful (e.g., have more practical interest), which have this property. In the next part of this section we give a necessary and sufficient condition for a distance based on a neighbourhood sequence to be a (generalized) metric (and we continue this analysis in [12], too).

Lemma 9. Let $p$ and $q$ be arbitrary sequences in $\mathbb{R}^{\infty}$, and let the neighbourhood sequence $B_{1}$ be faster than the neighbourhood sequence $B_{2}$ (i.e., $B_{1} \sqsupseteq^{*} B_{2}$ ). If there is no $B_{1}$-path between $p$ and $q$, then there does not exist any $B_{2}$-path between them.

Proof. From Definition 10, if $d\left(p, q ; B_{1}\right)=\infty$ and $B_{1}$ is faster than $B_{2}$, then $d\left(p, q ; B_{2}\right)=\infty$.

We show a property in which the distances based neighbourhood sequences differ in $\mathbb{Z}^{\infty}$ and in $\mathbb{R}^{\infty}$. 
Proposition 6. Let $p, q, r \in \mathbb{R}^{\infty}$ such a way that $p(i)<r(i)<q(i)$. If $p, q, r$ contain only integer elements, then the following statements hold (with their differencesequences):

$$
u_{p r}(i)+u_{r q}(i)=u_{p q}(i)
$$

because

$$
w_{p r}(i)+w_{r q}(i)=w_{p q}(i)
$$

and $w_{p r}(i)=u_{p r}(i), w_{r q}(i)=u_{r q}(i)$ and $w_{p q}(i)=u_{p q}(i)$ for all $i$, since

$$
(p(i)-r(i))+(r(i)-q(i))=p(i)-q(i) .
$$

For non integer valued sequences it is easy to show a counterexample.

The next theorem is the extension of the results about metrical properties of [10] (for previous results, see [5], concerning periodic neighbourhood sequences in finite dimension), to the arbitrary sequences. To formulate our result we need only the simple concepts of the faster relation and the shifted sequence (similarly to the case of $\mathbb{Z}^{\infty}$, as it is shown in [10]). We have to care only the triangle inequality, which is a statement for three sequences and we showed in Proposition 6 that the case of $\mathbb{Z}^{\infty}$ is not the same as $\mathbb{R}^{\infty}$. (There is no problem with the symmetry by Lemma 4 ) Based on the proof of the case $\mathbb{Z}^{\infty}$ using the properties of uids sequences the next theorem can be established.

Theorem 3. The distance function based on an neighbourhood sequence $B$ generates a generalized metric space on the set $\mathbb{R}^{\infty}$, if and only if $B(i)$ is faster than $B$ for all $i \in \mathbb{N}$.

(This theorem is proven in a more general form in the second part of the paper, see [12].)

By Lemma 7 one can decide, whether an neighbourhood sequence give a generalized metric space or not:

Proposition 7. The B-distance is a generalized metric if and only if

$$
\begin{gathered}
d(p, q ; B(i)) \leq d(p, q ; B), \quad \text { for all } p, q \in \mathbb{R}^{\infty} \text { and } i \in \mathbb{N} \text {, i.e., } \\
\qquad \sum_{j=1}^{m} b_{i+j}^{k} \geq \sum_{j=1}^{m} b_{j}^{k}, \quad \text { for all } i, k, m \in \mathbb{N} .
\end{gathered}
$$

By using the definition of the $k$-limited sequence it is equivalent to the condition

$$
\sum_{j=1+i}^{m+i} \min \left(b_{j}, k\right) \geq \sum_{j=1}^{m} \min \left(b_{j}, k\right), \quad \text { for all } i, k, m \in \mathbb{N} .
$$

Now we study the structure of the set of neighbourhood sequences based on another kind of partial ordering (see [7]). 
Definition 13. For $B=\left(b_{i}\right)_{i=1}^{\infty}, B^{\prime}=\left(b_{i}^{\prime}\right)_{i=1}^{\infty}$ we write $B \sqsupseteq B^{\prime}$ if and only if $b_{i} \geq b_{i}^{\prime}$ for every $i \in \mathbb{N}$.

It turns out that $\sqsupseteq$ has much more pleasant properties than $\beth^{*}$. Actually, this relation is proper part of the relation 'faster' (and in a certain sense, it is much stricter).

Remark 6. Let $B$ be an neighbourhood sequence. Then for the limited neighbourhood sequences of $B$ the following relations hold: $B \sqsupseteq B^{k} \sqsupseteq B^{m}$ for every $k, m \in \mathbb{N}$ with $k>m$.

We will use this new partial ordering relation to get lattice on the set of neighbourhood sequences, as the next statement shows.

Theorem 4. The set of neighbourhood sequences is a distributive lattice using the relation $\sqsupseteq$, with greatest lower bound $(1)_{i=1}^{\infty}$ and least upper bound $(\infty)_{i=1}^{\infty}$. Moreover, this lattice is complete.

Proof. The proof goes in the same way as in [7] for $\infty$ D neighbourhood sequences in $\mathbb{Z}^{\infty}$.

See [7] for other results using the relation $\sqsupseteq$ and $\sqsupseteq^{*}$ among special subsets of the neighbourhood sequences. Their properties are independent of their using conditions, therefore we do not give more details on them here. Instead of it, we are going to show another type of extensions of these distances in [12] as a continuation of this paper.

\section{ACKNOWLEDGEMENT}

The author is grateful to Zs. Páles, Z. Boros and A. Gilányi for their valuable remarks.

\section{REFERENCES}

[1] M. Borelli and A. Sgarro, "A possibilistic distance for sequences of equal and unequal length." Discrete Math. Theor. Comput. Sci., vol. Finite versus infinite, pp. 27-38, 2000.

[2] D. Cohn, Measure theory. Boston, Mass.: Birkhäuser, 1980.

[3] I. Csiszár and J. Körner, Information theory. Coding theorems for discrete memoryless systems. Probability and Mathematical Statistics. New York, London: Academic Press, 1981.

[4] P. Das, "Lattices of octagonal distances in digital geometry." Pat. Recog. Let., vol. 11, pp. 663667, 1990.

[5] P. Das, P. Chakrabarti, and B. Chatterji, "Distance functions in digital geometry." Inform. Sci., vol. 42, pp. 113-136, 1987.

[6] P. Das, P. Chakrabarti, and B. Chatterji, "Generalized distances in digital geometry." Inform. Sci., vol. 42, pp. 51-67, 1987.

[7] A. Fazekas, A. Hajdu, and L. Hajdu, "Lattice of generalized neighbourhood sequences in $n \mathrm{D}$ and $\infty$ D." Publ. Math. Debrecen, vol. 60, no. 3-4, pp. 405-427, 2002.

[8] K. Knopp, Theory and application of infinite series. London: Blackie \& Son Ltd., 1928, (translation: 1951). 
[9] B. Levitan and V. Zhikov, Almost periodic functions and differential equations. Cambridge, New York: Cambridge University Press, 1982.

[10] B. Nagy, "Distance functions based on neighbourhood sequences." Publ. Math. Debrecen, vol. 63, no. 3, pp. 483-493, 2003.

[11] B. Nagy, "Distances with generalized neighbourhood sequences in $n \mathrm{D}$ and $\infty \mathrm{D}$." Disc. Appl. Math., vol. 156, pp. 2344-2351, 2008, doi: 10.1016/j.dam.2007.10.017.

[12] B. Nagy, "Generalized distances of sequences II: $B$-distances with weight sequences," (submitted for publication).

[13] A. Rosenfeld and J. Pfaltz, "Distance functions on digital pictures." Pat. Recog., vol. 1, pp. 33-61, 1968.

[14] M. Yamashita and T. Ibaraki, "Distances defined by neighborhood sequences." Pat. Recog., vol. 19 , no. 3, pp. 237-246, 1986.

\section{Author's address}

\section{Benedek Nagy}

Eastern Mediterranean University, Faculty of Arts and Sciences, Department of Mathematics, Famagusta, North Cyprus, Mersin-10, Turkey

E-mail address: nbenedek. inf@gmail.com 